\title{
Outbreak investigation: molecular characterization of Vibrio cholerae isolates from Horn of Africa Lamyaa Shaban ${ }^{1}$, Salwa F Ahmed ${ }^{2}$, Sadiki Materu ${ }^{3}$ and John D Klena*2
}

\author{
Address: ${ }^{1}$ Biotechnology Graduate Program, American University-Cairo, Cairo, Egypt, ${ }^{2}$ US Naval Medical Research Unit-3, Cairo, Egypt and \\ ${ }^{3}$ African Medical and Research Foundation, Nairobi, Kenya \\ * Corresponding author
}

from 2009 American University in Cairo Research Conference

Cairo, Egypt. 5 April 2009

Published: I July 2009

BMC Proceedings 2009, 3(Suppl 3):O9

This abstract is available from: http://www.biomedcentral.com/I753-656I/3/S3/O9

(C) 2009 Shaban et al; licensee BioMed Central Ltd.

Vibrio cholerae has caused 7 global pandemics to date. The $7^{\text {th }}$ ongoing pandemic caused by serogroup $\mathrm{O} 1 \mathrm{El}$ Tor has been the most extensive in geographical spread and duration. It began in 1961 in Indonesia spreading through Asia and the Middle East and finally reaching Sub Saharan West Africa at the early 1970s, resulting in over 150,000 cases and more than 20,000 deaths during the first year. $V$. cholerae is currently responsible for several outbreaks in Africa, some with a reported high mortality rate. Although extensive characterization has been carried out on epidemic strains in Asia, little information is available regarding the characteristics of strains causing the recent outbreaks in Africa. The aim of the present study is to characterize at the genotypic level the different epidemiologic strains that are part of several outbreaks that occurred in Africa during 2007. Different molecular techniques are used in order to evaluate a reliable tool that can be used to relate/discriminate the $V$. cholerae isolates obtained from diverse geographical areas. This study will investigate whether a cluster of isolates or a dominant clone is responsible for the spread of the reported outbreaks. Eighty isolates of $V$. cholerae were phenotypically characterized using conventional microbiological procedures such as biochemical testing and antimicrobial resistance, and confirmed serologically by the slide agglutination test using specific antiserum to $V$. cholera polyvalent $\mathrm{O} 1$ as well as serotype specific antisera for Inaba and Ogawa subtypes. Molecular characterization of strains are performed by using polymerase chain reaction (PCR), multilocus variable tandem repeat analysis (MLVA), mulitlocus sequence typing (MLST), and DNA sequencing for several virulent genetic elements such as the cholera toxin. 